\title{
SUPPLEMENT TO: FUNCTIONAL FACTOR ANALYSIS FOR PERIODIC REMOTE SENSING DATA
}

\author{
By Chong Liu and Surajit Ray and Giles Hooker and Mark Friedl \\ Boston University and Cornell University
}

1. Data. Data from MODIS were extracted for a 25 by 25 pixel window (covering an area of $\approx 134 \mathrm{Km}^{2}$ ) centered over the Harvard Forest Long Term Experimental Research site in Petersham, MA. Data are provided at 8-day intervals with 500-m spatial resolution on the ground for the period from January 1, 2001 to December 31, 2006. The data set is available at Center for Remote Sensing public release data set server at Boston University Department of Geography.

1.1. Pre-Processing. Because EVI data are sensitive to the presence of snow and include noise and missing values caused by clouds, the data were pre-processed prior to analysis to remove noise and fill gaps following the procedure described by Zhang, Friedl and Schaaf (2006). Specifically, instances of missing data for single dates caused by clouds were gap-filled using a three-point moving average. To remove the effect of snow, which can significantly depress EVI values relative to snow-free conditions, we used flags included with MODIS data that indicate the presence of snow in any pixel. In cases where snow was present, EVI values were replaced with their most recent snow-free value. Finally, to remove outliers and reduce overall noise, a three-point median-value moving-window was applied to the time series at each pixel.

1.2. Problematic Pixels and their imputation. The preprocessed EVI in the raw data set which includes 625 pixels contains values which are suspected to be too low for the vegetation type at Harvard Forest. We set threshold at 0.1 which corresponds to the historical minimum EVI values under snow-free conditions at this site. Any pixels with any EVI values below this threshold is labeled as problematic. In addition, we delete observations below the threshold in the raw data set and treat them as missing values. The resulted missingness at each problematic pixel occurs in long sequences, so that direct smoothing on individual curves cannot be done. After excluding problematic pixels, the final data set consists of 423 pixels and each pixel has 276 EVI values observed at equally spaced time grids. These observations, denoted by $\left\{Y_{i j}, 1 \leq i \leq 423,1 \leq j \leq 276\right\}$ are of good quality and contain no missing values. We denote observations at problematic pixels by $\left\{Y_{i j}^{*}, 1 \leq i \leq 202,1 \leq j \leq n_{i}\right\}$.

For the purpose of examining spatial structure in the PPC scores, we impute $Y_{i j}^{*}$ 's and reconstruct whole EVI curves at these pixels. To this end, we use a variant of the Principal Analysis with Conditional Expectation (PACE) procedure introduced by Yao, Müller and Wang (2005). The PACE allows one to impute sparsely observed functional data, provided that we know or can estimate the functional covariance structure.

As a brief overview of PACE, recall that we consider EVI data as discrete observations of an underlying $L^{2}$ stochastic process $X(t)$ with additive errors. Let $x_{i}(t)$ be the realization of $X(t)$ at pixel $i$. In the paper, we denote the estimate of $x_{i}(t)$ by $\hat{x}_{i}(t)$, the time-series 
demeaned $\hat{x}_{i}(t)$ by $z_{i}(t)$ and cross-section demeaned $z_{i}(t)$ by $\tilde{z}_{i}(t)$. We model observed data as $Y_{i j}=x_{i}\left(t_{i j}\right)+e_{i j}$ and $Y_{i j}^{*}=x_{i}^{*}\left(t_{i j}\right)+e_{i j}^{*}$, where $e_{i j}$ 's and $e_{i j}^{*}$ 's are i.i.d. observational errors with mean zero and variance $\sigma^{2}$. They are independent with both $x_{i}(t)$ 's and $x_{i}^{*}(t)$ 's. Given $Y_{i j}$ 's, we estimate $x_{i}(t)$ by smoothing $Y_{i j}$ with $K=277$ Fourier basis functions. Then the estimated covariance kernel $\hat{C}(s, t)=\widehat{\operatorname{cov}}(X(s), X(t))$ has the following eigen-decomposition $\hat{C}(s, t)=\sum_{k=1}^{K} \omega_{k} \phi_{k}(s) \phi_{k}(t)$ and each $\hat{x}_{i}(t)$ can be represented as

$$
\hat{x}_{i}(t)=\hat{\mu}(t)+\sum_{k=1}^{K} s_{i k} \phi_{k}(t)
$$

where fPC scores $s_{i k}$ are independent for fixed $k$ and uncorrelated for fixed $i$ with mean zero and variance $\omega_{k}$. Note the above fPCA differs from the techniques employed in the paper where fPCA is employed explicitly on time-series demeaned process $Z(t)=X(t)-\int X(t) d t$.

To impute $Y_{i j}^{*}$ and estimate $x_{i}^{*}(t)$, direct smoothing might not be appropriate due to the existence of consecutive missing values. On the other hand, it is not appropriate either to impute the entire raw data set by smoothing covariance surface as described in Yao, Müller and Wang (2005), since a large amount of missingness occur at the same time period which does not satisfy the method's assumption. However, it is reasonable to assume $x_{i}^{*}(t)$ 's share the same eigen-structure and mean process with $x_{i}(t)$ 's. Then we have $\hat{x}_{i}^{*}(t)=\hat{\mu}(t)+\sum_{k=1}^{K} s_{i k}^{*} \phi_{k}(t)$. Estimating $x_{i}^{*}(t)$ reduces to estimating $\mathrm{fPC}$ score $s_{i k}^{*}$ and $\phi_{k}(t)$ can be computed from $\hat{x}_{i}(t)$. Writing $\boldsymbol{X}_{i}^{*}=\left(x_{i}^{*}\left(t_{i 1}\right), x_{i}^{*}\left(t_{i 2}\right), \cdots, x_{i}^{*}\left(t_{i n_{i}}\right)\right)^{T}, \boldsymbol{Y}_{i}^{*}=\left(Y_{i 1}^{*}, Y_{i 2}^{*}, \cdots, Y_{i n_{i}}^{*}\right)^{T}, \hat{\boldsymbol{\mu}}_{i}=$ $\left(\hat{\mu}\left(t_{i 1}\right), \hat{\mu}\left(t_{i 2}\right), \cdots, \hat{\mu}\left(t_{i n_{i}}\right)\right)^{T}, \boldsymbol{\varphi}_{i k}=\left(\phi_{k}\left(t_{i 1}\right), \phi_{k}\left(t_{i 2}\right), \cdots, \phi_{k}\left(t_{i n_{i}}\right)\right)^{T}$, the PACE result states that, the best prediction of $\mathrm{fPC}$ scores for the $i$ th subject, given data from that individual, is the conditional expectation, which under Gaussian assumption, can be shown to be

$$
\hat{s}_{i k}^{*}=\mathcal{E}\left(s_{i k}^{*} \mid \boldsymbol{Y}_{i}^{*}\right)=\omega_{k} \boldsymbol{\varphi}_{i k}^{T}\left(\boldsymbol{\Sigma}_{i}+\sigma^{2} \boldsymbol{I}_{n_{i}}\right)^{-1}\left(\boldsymbol{Y}_{i}^{*}-\hat{\boldsymbol{\mu}}_{i}\right),
$$

where the $j k$ th entry of $\boldsymbol{\Sigma}_{i}$ is given by $\hat{C}\left(t_{i j}, t_{i k}\right)$ for $1 \leq j, k \leq n_{i} . \sigma^{2}$ can be estimated as the sample variance of $\left\{\hat{e}_{i j}=Y_{i j}-\hat{x}_{i}\left(t_{i j}\right), 1 \leq i \leq 423,1 \leq j \leq 276\right\}$. In fact, this result is the conditional mean of two jointly Gaussian random variables. It can be shown that, under Gaussian assumption, $\operatorname{Cov}\left(\boldsymbol{Y}_{i}^{*}\right)=\boldsymbol{\Sigma}_{i}+\sigma^{2} \boldsymbol{I}_{n_{i}}$ and $\left.\operatorname{Cov}\left(s_{i k}^{*}, \boldsymbol{Y}_{i}^{*}\right)\right)=\omega_{k} \boldsymbol{\varphi}_{i k}^{T}$.

Denote the first fPC of $z_{i}(t)$ 's by $\gamma_{1}(t)$, as in the paper and the first fPC of the non-annual component of $z_{i}(t)$ 's by $\eta_{1}(t)$. Denote time-series demeaned $\hat{x}_{i}^{*}(t)$ by $z_{i}^{*}(t)$ and cross-section demeaned $z_{i}^{*}(t)$ by $\tilde{z}_{i}^{*}(t)$. Then fPC scores of $z_{i}^{*}(t)$ on $\gamma_{1}(t)$ and $\eta_{1}(t)$ are computed as follows,

$$
\begin{aligned}
& s_{i 1}^{* \gamma}=\int \tilde{z}_{i}^{*}(t) \gamma_{1}(t) d t \\
& s_{i 1}^{* \eta}=\int \tilde{z}_{i}^{*}(t) \eta_{1}(t) d t
\end{aligned}
$$

Combine imputed scores $s_{i 1}^{* \gamma}$, s and $s_{i 1}^{* \eta}$, s together with scores computed from good pixels, score maps of 625 pixels can be formed which are shown in Figure 1. Score maps without imputing problematic pixels are also shown in Figure 1 for comparison.

As a concluding analysis for this section, we compare a set of results obtained in the paper using all 625 pixels with problematic ones imputed following procedures discussed above against the same analysis leaving out the problematic pixels. Figure 2 shows that the results are very similar. 

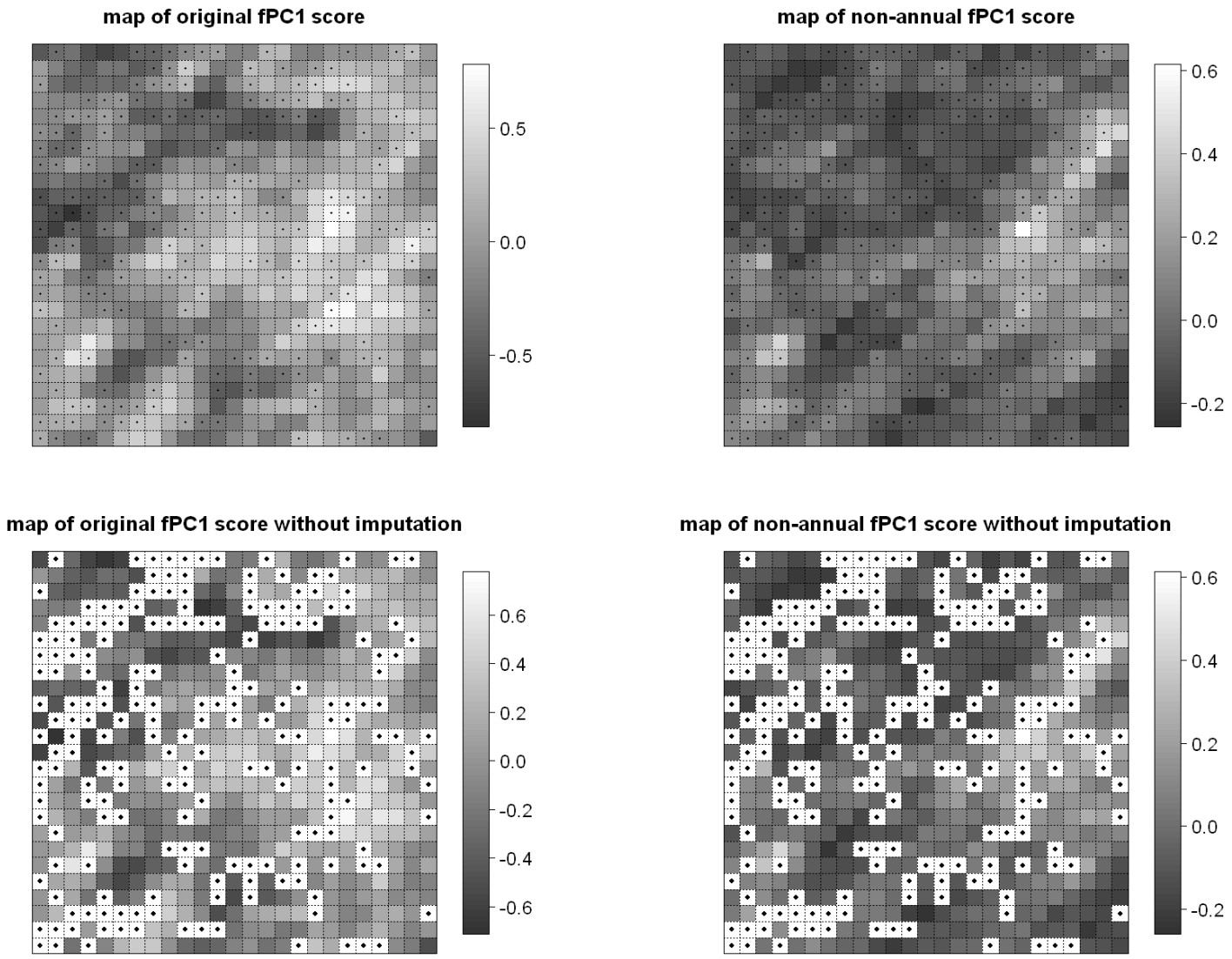

FIG 1. In all plots, dotted squares represent problematic pixels. Upper left: scores on the first original fPC. $f P C$ scores on problematic pixels are obtained by projecting imputed EVI curves onto the original fPC obtained from good pixels; Upper right: scores on the first non-annual fPC. fPC scores on problematic pixels are obtained by projecting imputed EVI curves onto the non-annual fPC obtained from good pixels. Lower left: the first original $f P C$ scores without imputing the problematic pixels; Lower right: the first non-annual fPC scores without imputing the problematic pixels.

2. Annual Information. AI is computed as the cumulative variation explained by benchmarks as a proportion of that explained by PPCs. In the paper, we propose using AI to graphically facilitate the labeling of PPCs as either nearly-annual or non-annual, by plotting AI against cumulative variation explained by PPCs. In this section, we design a simulation to demonstrate the efficiency of this technique. The main idea is to construct a collection of eigenfunctions which include both nearly-annual and non-annual functions.

Particularly, we use 40 Fourier basis functions on interval $[0, T]$ to construct 40 eigenfunctions which all have zero time-series mean. Let $\left\{\varphi_{j}, 1 \leq j \leq 40\right\}$ be the Fourier basis functions and $\boldsymbol{\varphi}$ be the column vector of $\varphi_{j}$ 's. Let $\left\{\gamma_{k}, 1 \leq k \leq 40\right\}$ be eigenfunctions and $\gamma$ be the column vector of $\gamma_{k}$ 's. let $\left\{\lambda_{k}, 1 \leq k \leq 40\right\}$ be corresponding eigenvalues and $\omega$ be the basic 

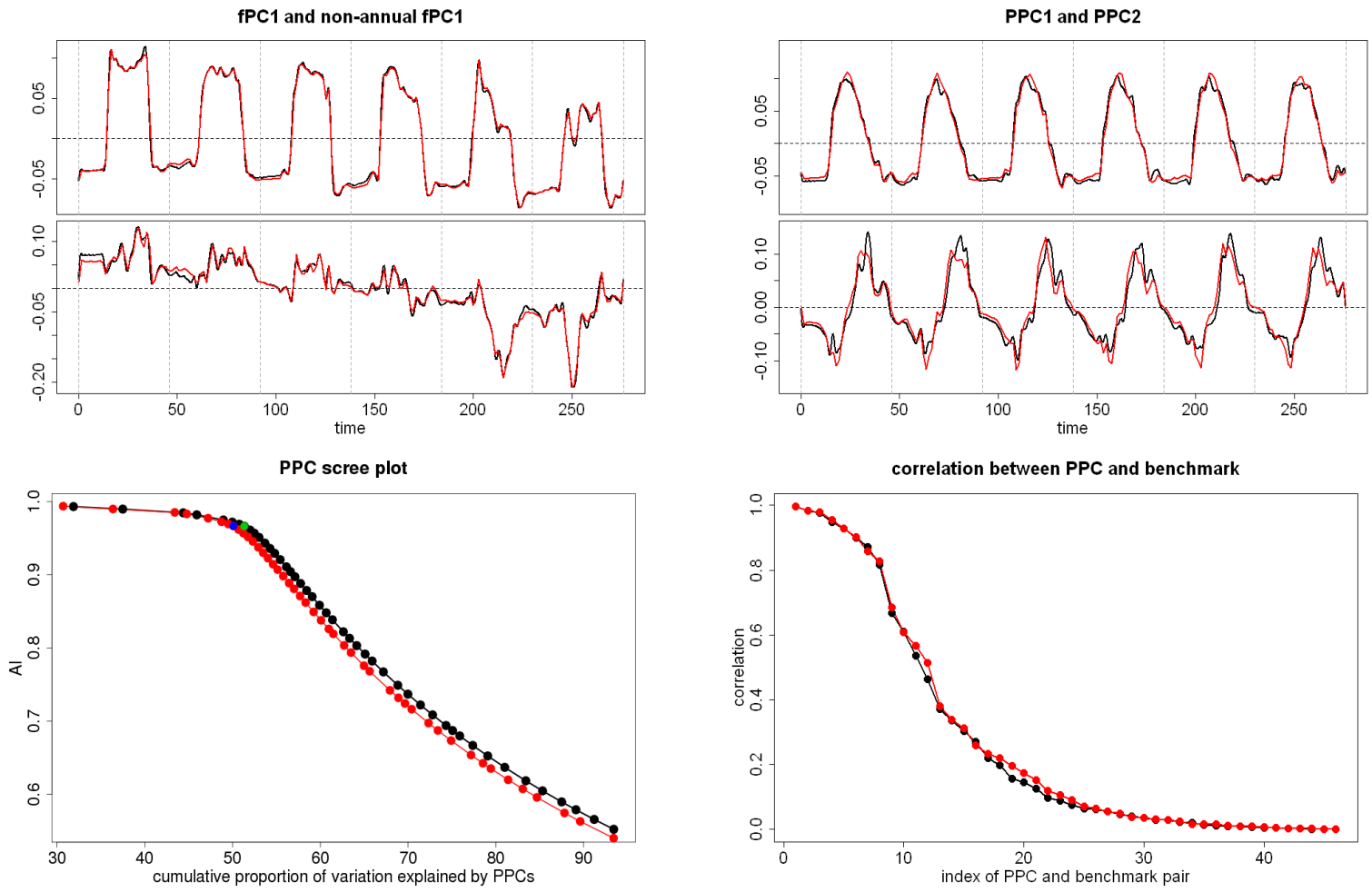

FIG 2. In all four plots, black curves are obtained from 423 good pixels and red curves are obtained from 625 pixels with problematic ones imputed from eigen-structure of the good pixels. Upper left: the upper panel is the first original $f P C$ and the lower panel is the first non-annual fPC; Upper right: the upper panel is the first PPC and the lower panel is the second PPC. Both black and red curves are obtained by rotating the first 46 fPCs; Lower left: PPC scree plot. The green dot is the 8th AI on the black curve and the blue dot is the 8th $A I$ on the red curve; Lower right: correlations between PPCs and associated benchmarks.

angular frequency. Then

$$
\begin{aligned}
& \varphi_{j}=\sin \left(\frac{j+1}{2} \omega t\right), \text { if } j \text { is odd } \\
& \varphi_{j}=\cos \left(\frac{j}{2} \omega t\right), \text { if } j \text { is even }
\end{aligned}
$$

Suppose $[0, T]$ spans over 4 complete cycles, or years. Let $A=\{j \mid j=8 a-1$, or $j=8 a, 1 \leq$ $a \leq 5\}$. Then $\varphi_{A}=\left\{\varphi_{j} \mid j \in A\right\}$ is the collection of annual Fourier basis functions. Denote the space spanned by $\varphi_{A}$ by $\mathbf{F}_{P}$.

Let $\mathbf{C}$ be the coefficient matrix of eigenfunctions. Then $\gamma=\mathbf{C} \boldsymbol{\varphi}$. In constructing $\gamma$, one could simply let $\mathbf{C}=\mathbf{I}$. Then, $\gamma=\boldsymbol{\varphi}$. Assuming the retained fPC space $\boldsymbol{\Gamma}_{M}$ contains at least one function in $\boldsymbol{\varphi}_{A}$, then $\mathbf{F}_{P}$ and $\boldsymbol{\Gamma}_{M}$ have a common subspace and any pair of orthogonal rotations on $\mathbf{F}_{P}$ and $\boldsymbol{\Gamma}_{M}$ which share the same operation on this common subspace will yield a valid PPC result with correlations between all pair of PPCs and benchmarks in the common subspace equal to 1 . This is a special case where we lose the uniqueness of PPC 
rotation which is not desirable in simulation and moreover, this case is rarely possible to occur in real practice. Therefore, we add a perturbation to the identity matrix and obtain $\hat{\mathbf{C}}=\mathbf{I}+\sigma_{p} \boldsymbol{\epsilon}$, where $\boldsymbol{\epsilon}$ is a random matrix of the same size as $\mathbf{C}$ and $\left\{\boldsymbol{\epsilon}_{i j}, 1 \leq i, j \leq 40\right\}$ are i.i.d. Gaussian distributed with mean zero and variance 1. $\sigma_{p}$ is called the perturbation standard deviation controlling how far eigenfuncions $\gamma$ deviate from $\varphi$. Note $\gamma$ needs to be an orthogonal system. Hence, the Gram-Schmidt orthonormalization is performed on $\hat{\mathbf{C}}$ to obtain $\mathbf{C}$. Among all rows of $\hat{\mathbf{C}}$, row indices included in set $A$ are orthonormalized first and within $A$, row with smaller index is orthonormalized first.

In the simulation, we set $\sigma_{p}=0.1$. In this way, we construct 40 eigenfunctions within which, $10(|A|=10)$ are nearly-annual and the other 30 are nearly-non-annual. Given these eigenfunctions, we can construct stochastic process $X(t)$ as follows

$$
X(t)=\sum_{k=1}^{40} s_{k}^{\gamma} \gamma_{k}(t)
$$

where $\left\{s_{k}^{\gamma}, 1 \leq k \leq 40\right\}$ are uncorrelated Gaussian random variables. For the purposes of this study we treat the true curves for each realization as being observed exactly and thus add no extra errors onto the smooth curves. In this simulation, we examine 4 sets of simulated curves which include different number of nearly-annual eigenfunctions. Denote the $i$ th realization of $s_{k}^{\gamma}$ for $j$ th curve set by $s_{i j k}^{\gamma}$. Then, $s_{i j k}^{\gamma}$ 's are i.i.d. Gaussian distributed with mean zero and variance $\lambda_{j k}$. Let $A_{1: n}$ be the set of the first $\mathrm{n}$ smallest indices in $A$. Then simulated curves are generated as,

$$
\begin{aligned}
x_{i}^{j}(t) & =\sum_{k=1}^{40} s_{i j k}^{\gamma} \gamma_{k}(t), 1 \leq j \leq 4,1 \leq i \leq 400, \text { with } \\
\lambda_{j k} & =1, \text { if } k \in A^{c} \\
\lambda_{j k} & =6 /(j+1), \text { if } k \in A_{1:(j+1)} \\
\lambda_{j k} & =0, \text { otherwise. }
\end{aligned}
$$

In (1), we have $j+1$ nearly-annual eigenfunctions in the $j$ th set of simulated curves $\left\{x_{i}^{j}(t), 1 \leq\right.$ $i \leq 400\}$. Note that we keep the total variation on nearly-annual eigenfunctions to be 6 across $j$, which accounts for $20 \%$ of that explained by nearly-non-annual eigenfunctions. In each set of simulated data, we have 400 curves and PPC computation can produce a single scree plot. We expect to observe a kink at $(j+1)$ th $\mathrm{AI}$ in the scree plot for the $j$ th set of data. To verify this, we repeat the procedure in (1) for $B=500$ times and overlay 500 scree plots together for each $j$. In computing PPCs, we fix the percentage of variation explained at $90 \%$ for all $j$ 's. The results are shown in Figure 3 where we observe that the desired number of periodic components is chosen with very high probability.

3. Bootstrap hypothesis test. In this section, we discuss some results related to the bootstrap hypothesis testing procedure proposed in the paper. We first examine the effect of pre-smoothing on our test and next, present testing results on Harvard Forest data. Finally, we describe simulation studies where we explore the empirical power curve and size on simulated data sets. 

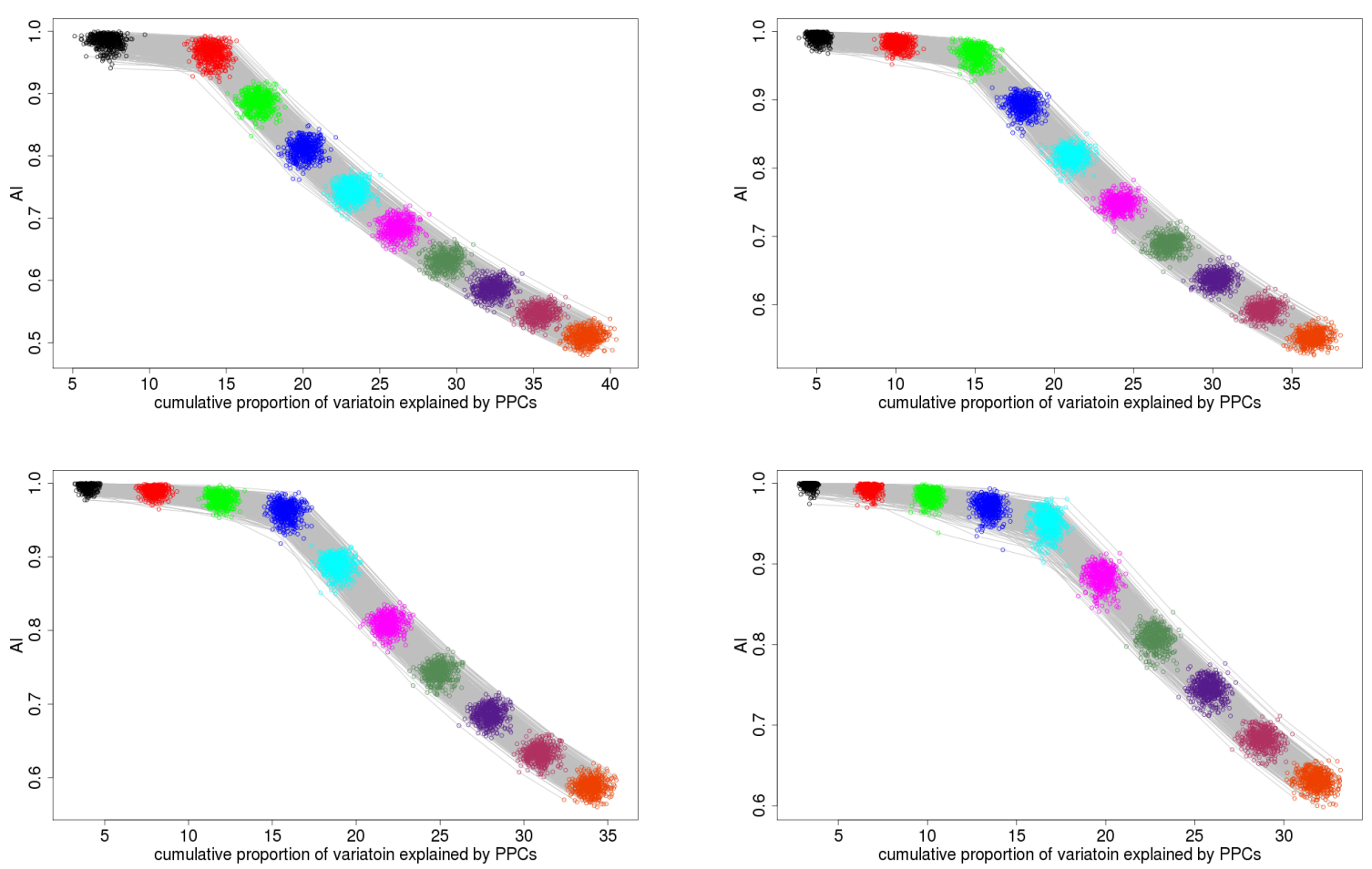

FIG 3. 500 scree plots overlaid for each j. Circles in different colors represent replicated AI's of different indices. Grey solid lines connect circles of different colors from the same replication. Upper left: 2 nearly-annual PPCs; Upper right: 3 nearly-annual PPCs; Lower left: 4 nearly-annual PPCs; Lower right: 5 nearly-annual PPCs

3.1. Bootstrap residuals. It has been noted that the choice of smoothing parameter can affect the proposed hypothesis test. Yao, Müller and Wang (2005) showed that the sampling variability due to pre-smoothing can be ignored asymptotically if we work in the case of dense observations. However, given the finite sample at hand, it is still worth examining the sampling variability due to pre-smoothing compared with the variability due to bootstrapping pre-smoothed curves. If the former variability is not negligible compared to the later one, it is not appropriate to simply bootstrap pre-smoothed curves in constructing the null distribution of the test statistic. Given pre-smoothed curves $\hat{x}_{i}(t)$ 's, we know estimated residuals $\hat{\epsilon}_{i j}=$ $Y_{i j}-\hat{x}_{i}\left(t_{i j}\right), 1 \leq i \leq 423,1 \leq j \leq 276$. Then the $b$ th bootstrap residuals $\left\{\hat{\epsilon}_{i j}^{b}, 1 \leq i \leq\right.$ $423,1 \leq j \leq 276\}$ are obtained by sampling with replacement from $\hat{\epsilon}_{i j}$ 's pooled across $i$ and $j$. Then the $b$ th bootstrap EVI observations $\left\{Y_{i j}^{b r}, 1 \leq i \leq 423,1 \leq j \leq 276\right\}$ are formed by

$$
Y_{i j}^{b r}=\hat{x}_{i}\left(t_{i j}\right)+\epsilon_{i j}^{b}
$$

where the superscript $b r$ stands for bootstrap residuals. Suppose we bootstrap $B$ times. We re-smooth each bootstrap EVI observations with optimal smoothing parameters chosen by the sum of generalized cross validation scores across all pixels and obtain smoothed curves $\left\{\hat{x}_{i}^{b r}(t), 1 \leq b \leq B, 1 \leq i \leq 423\right\}$. On the other hand, we directly sample with replacement 
from $\hat{x}_{i}(t)$ 's and form the $b$ th direct bootstrap curves, denoted $\left\{\hat{x}_{i}^{b c}(t), 1 \leq i \leq 423\right\}$ where the superscript $b c$ stands for bootstrap curves. Then, we compute PPCs on both $\left\{\hat{x}_{i}^{b r}(t), 1 \leq\right.$ $i \leq 423\}$ and $\left\{\hat{x}_{i}^{b c}(t), 1 \leq i \leq 423\right\}$ for each $b$. The variability of the first PPCs from 1000 bootstrap samples are summarized in Figure 4 . Note that it is more exact to bootstrap $z_{i}(t)$ 's rather than $\hat{x}_{i}(t)$ 's. However, it makes no difference in results since $z_{i}(t)$ and $\hat{x}_{i}(t)$ are identical up to a vertical shift and we take out this vertical shift when computing PPCs. In subsequent sections where we discuss the hypothesis test procedure, bootstrapping is based on time-series demeaned curves $z_{i}(t)$ 's.
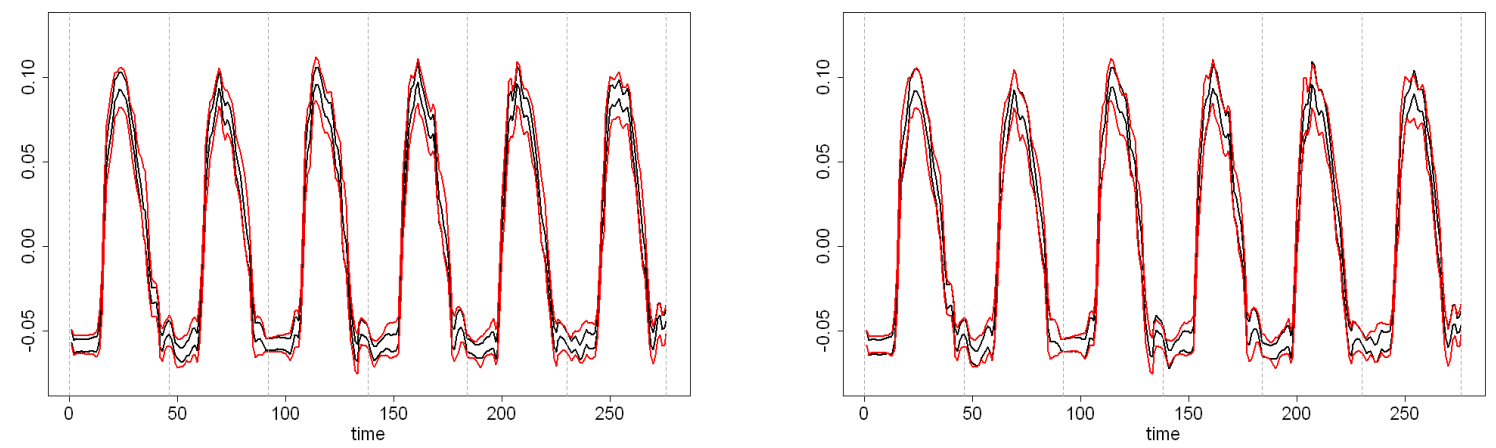

FIG 4. In both plots, red curves are lower and upper 0.025 pointwise confidence interval of the first PPCs obtained by directly bootstrapping pre-smoothed curves 1000 times, i.e. PPCs of $\left\{\hat{x}_{i}^{b c}(t), 1 \leq i \leq 423\right\}$. Black curves are lower and upper 0.025 pointwise confidence interval of the first PPCs obtained by bootstrapping residuals 1000 times with curves held constant, i.e. PPCs of $\left\{\hat{x}_{i}^{b r}(t), 1 \leq i \leq 423\right\}$. Left: pointwise confidence interval derived by fixing the number of fPCs retained when computing PPCs; Right: pointwise confidence interval derived by fixing the percentage of variation explained when computing PPCs

The variability due to smoothing is smaller than that of bootstrapping pre-smoothed curves but still not negligible. Therefore, it is more appropriate to add bootstrap residuals onto the bootstrap pre-smoothed curves in the hypothesis testing procedure.

3.2. Hypothesis testing. As described in the paper, we propose one way of constructing approximated null hypothesis through the replacement of the first PPC by its benchmark and construct $\bar{z}_{i}(t)$ 's. Next, analysis in Section 3.1 suggests we bootstrap $\bar{z}_{i}(t)$ 's with residuals. In particular, the $b$ th bootstrap null curves $\left\{\bar{z}_{i}^{b}(t), 1 \leq i \leq 423\right\}$ are formed by sampling with replacement from $\bar{z}_{i}(t)$ 's. We also sample with replacement from estimated residuals $\hat{e}_{i j}$ 's and form the $b$ th bootstrap residuals $\left\{\hat{e}_{i j}^{b}, 1 \leq i \leq 423,1 \leq j \leq 276\right\}$. Then, the $b$ th bootstrap observations are constructed as

$$
W_{i j}^{b}=\bar{z}_{i}^{b}\left(t_{i j}\right)+\hat{e}_{i j}^{b}, 1 \leq i \leq 423,1 \leq j \leq 276
$$

We re-smooth $W_{i j}^{b}$ 's for each $b$, compute PPCs either by fixing the number of fPCs or the percentage of variation, and then calculate bootstrap correlation $\rho_{1}^{b}$. The histogram of $\left\{\rho_{1}^{b}, 1 \leq b \leq B\right\}$ under two formulations of null hypothesis are shown in Figure 5 . This proposed bootstrap testing procedure is the same in principal as that introduced in Li and 
Chiou (2011), where authors tested the equality of functional means and covariances of two clusters.

The second way of approximating null hypothesis rescales PPC scores after the replacement and construct $\check{z}_{i}(t, \hat{\boldsymbol{\tau}})$ where $\hat{\boldsymbol{\tau}}$ is the optimal rescaling vector. Before doing bootstrap, we need to find the optimal rescaling vector $\boldsymbol{\tau}$. To reduce computation time, we place only two rescaling parameters $\tau_{1}$ on the first benchmark and $\tau_{2}$ on all remaining PPCs. Intuitively, rescaling by only two parameters yield more conservative null compared to using 46 parameters; this could be tested by introducing more degrees of freedom for the $\tau_{j}$. The hypothesized curves are formed by

$$
\check{x}_{i}\left(t, \tau_{1}, \tau_{2}\right)=\tau_{1} s_{i 1}^{\xi} \theta_{1}(t)+\tau_{2} \sum_{j=2}^{M} s_{i j}^{\xi} \xi_{j}(t)+\sum_{k=M+1}^{K} s_{i j}^{\gamma} \gamma_{j}(t),
$$

and we need to solve the following optimization problem

$$
\begin{aligned}
& \min _{\tau_{1}, \tau_{2}} K L\left(\boldsymbol{\Omega}_{0}, \boldsymbol{\Omega}\right)-\lambda \log \check{\rho}_{1}\left(\tau_{1}, \tau_{2}\right) \\
= & \frac{1}{2}\left(\tau_{1}^{2}-1-\log \tau_{1}^{2}\right)+\frac{M-1}{2}\left(\tau_{2}^{2}-1-\log \tau_{2}^{2}\right)-\lambda \log \check{\rho}_{1}\left(\tau_{1}, \tau_{2}\right) .
\end{aligned}
$$

(3) is solved by Nelder-Mead method with initial values $\tau_{1}=\tau_{2}=1$, and a sequence of $\lambda$ values. The results are summarized in Table 1 . When fixing number of fPCs retained, the

\begin{tabular}{ccccccc}
\hline$\lambda$ & $\tau_{1}^{n}$ & $\tau_{2}^{n}$ & $\rho_{1}^{n}$ & $\tau_{1}^{p}$ & $\tau_{2}^{p}$ & $\rho_{1}^{p}$ \\
\hline no rescaling & 1.000000 & 1.000000 & 0.9999984 & 1.000000 & 1.000000 & 0.9999984 \\
1 E4 & 1.014435 & 1.000303 & 0.9999985 & 1.014932 & 1.000379 & 0.9999985 \\
5 E4 & 1.061538 & 1.001336 & 0.9999988 & 1.050367 & 1.000045 & 0.9999987 \\
1 E5 & 1.102428 & 1.001916 & 0.9999990 & 1.051332 & 0.999486 & 0.9999987 \\
5 E5 & 1.264750 & 1.004882 & 0.9999994 & 1.056739 & 0.996366 & 0.9999987 \\
1 E6 & 1.366295 & 1.006796 & 0.9999996 & 1.057842 & 0.995725 & 0.9999987 \\
5 E6 & 1.686579 & 1.012825 & 0.9999998 & 1.058459 & 0.995366 & 0.9999987 \\
1 E7 & 1.855772 & 1.016094 & 0.9999999 & 1.058526 & 0.995327 & 0.9999987 \\
$5 \mathrm{E} 7$ & 2.356882 & 1.026565 & $\approx 1$ & 1.058560 & 0.995308 & 0.9999987 \\
\hline
\end{tabular}

TABLE 1

Optimal $\tau_{1}, \tau_{2}$ and corresponding $\rho\left(\tau_{1}, \tau_{2}\right) . \tau_{1}^{n}, \tau_{2}^{n}$ and $\rho_{1}^{n}$ represent solutions obtained by fixing the number of fPCs retained when computing PPCs. $\tau_{1}^{p}, \tau_{2}^{p}$ and $\rho_{1}^{p}$ represents solutions obtained by fixing the percentage of variation explained when computing PPCs.

difference between computed $\rho_{1}$ and 1 decreases to less than $10^{-6}$ at $\lambda=1$ E5. On the other hand, the first correlation stabilizes also at $\lambda=1 \mathrm{E} 5$ when fixing the percentage. For illustration, we choose $\lambda=1$ E5 for both cases of fixing number and percentage. Denote the optimal $\tau_{1}$ and $\tau_{2}$ by $\hat{\tau}_{1}$ and $\hat{\tau}_{2}$, we can construct hypothesized curves $\left\{\check{z}_{i}\left(t, \hat{\tau}_{1}, \hat{\tau}_{2}\right), 1 \leq i \leq\right.$ $423\}$ according to $(2)$. Then we bootstrap $\check{z}_{i}\left(t, \hat{\tau}_{1}, \hat{\tau}_{2}\right)$ 's and add bootstrap residuals. The $b$ th bootstrap observations are formed as

$$
W_{i j}^{b}=\check{z}_{i}^{b}\left(t, \hat{\tau}_{1}, \hat{\tau}_{2}\right)+\hat{e}_{i j}^{b}, 1 \leq i \leq 423,1 \leq j \leq 276
$$

The histogram of the bootstrap correlation computed from re-smoothed $W_{i j}^{b}$ 's for $1 \leq b \leq B$ under two formulations of the null hypothesis are shown in Figure 5. It is observed that little 
difference is made when inflating PPC scores with fixed percentage of variation explained. Note inflation causes more variation concentrated on the first benchmark. By retaining same amount of variation, less fPCs might be retained and thus there are less flexibilities in representing annual signals.
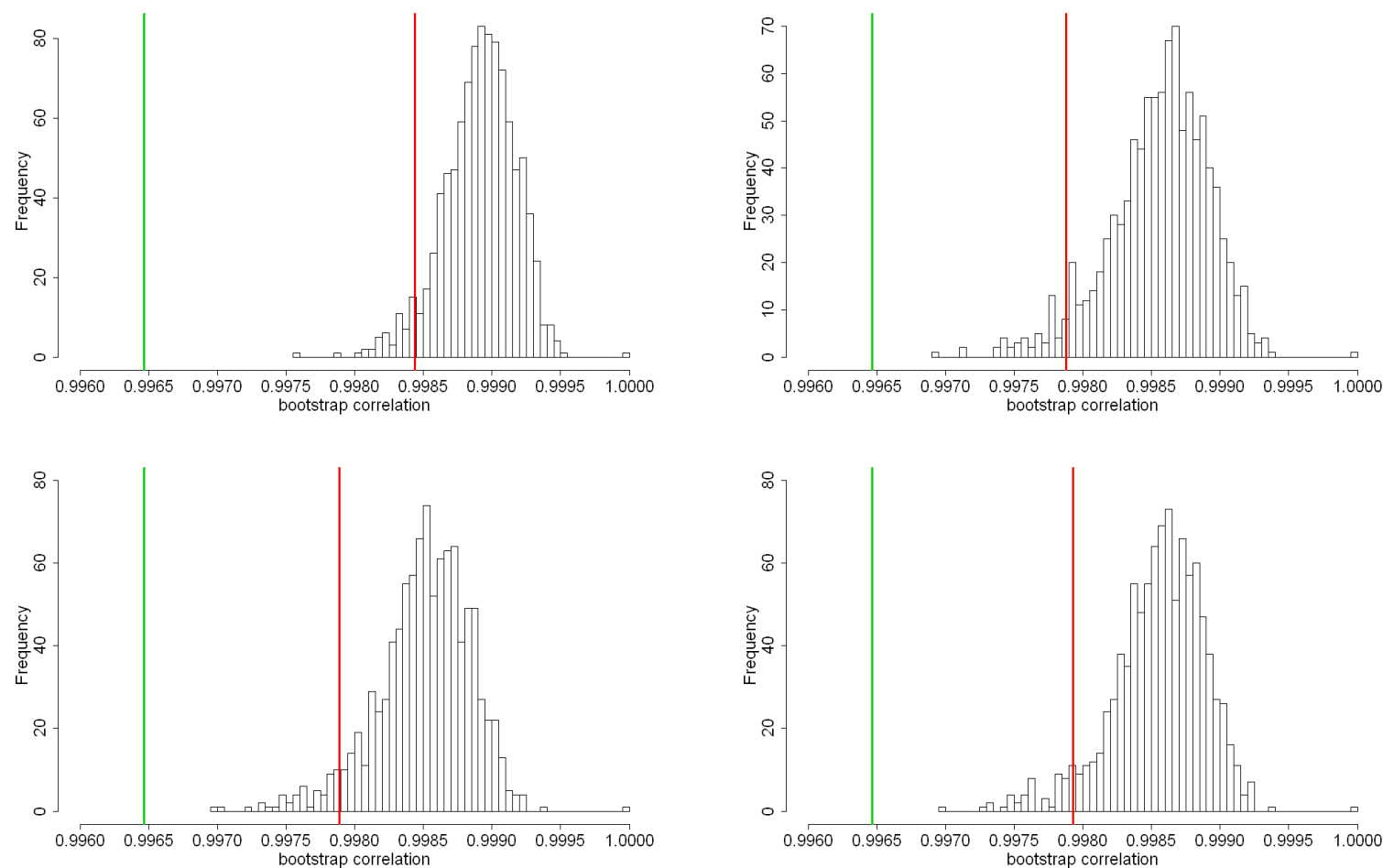

FIG 5. In all four plots, red solid lines are lower 0.05 critical values and green solid lines are the first correlation computed from Harvard Forest data. Upper left: histogram of bootstrap first correlation obtained by both replacement and inflation with fixed number of fPCs retained; Upper right: histogram of bootstrap first correlation obtained by both replacement and inflation with fixed percentage of variation explained; Lower left: histogram of bootstrap first correlation obtained by only replacement with fixed number of fPCs retained; Lower right: histogram of bootstrap first correlation obtained by only replacement with fixed percentage of variation explained.

3.3. Empirical power curve and size. In this section, we present a simulation study to examine the size and power of the test proposed in Section 3.7 of the paper. We examine a collection of alternative hypothesis which is characterized by a single parameter $\alpha$. Each alternative depends on a set of eigenfunctions. To describe the simulation design, we use the same notations introduced in Section 2. Given the Fourier basis functions $\varphi$ which are the same as those used in Section 2, we construct 36 eigenfunctions $\left\{\gamma_{k}^{\alpha}, 1 \leq k \leq 36\right\}$ and let $\gamma_{\alpha}$ be the column vector of $\gamma_{k}^{\alpha}$ 's. Thus, the $\alpha$ parametrized coefficient matrix $\mathbf{C}_{\alpha}$ now has size $36 \times 40$. Similar to the framework for the simulation in section 2 above, we add perturbation onto pre-chosen Fourier basis functions. However, the choice of template Fourier basis functions differs. Denote $T=\left\{j \mid j \in A_{1: 6}\right.$ or $\left.j \in A^{c}\right\}$. Then template functions 
in this simulation study are $\varphi_{T}=\left\{\varphi_{j} \mid j \in T\right\}$. Arrange $T$ by placing indices in $A_{1: 6}$ before $A^{c}$ where indices in $A_{1: 6}$ and $A^{c}$ are arranged by increasing order respectively. Denote the $i$ th index in $T$ after this arrangement by $T_{i}$. We can write the coefficient matrix of $\varphi_{T}$ as

$$
\begin{aligned}
& \mathbf{D}_{k j}=1, \text { if } j=T_{k} ; \\
& \mathbf{D}_{k j}=0, \text { otherwise; } \\
& 1 \leq k \leq 36 .
\end{aligned}
$$

We have $\boldsymbol{\varphi}_{T}=\mathbf{D} \boldsymbol{\varphi}$. Note the first 6 rows of $\mathbf{D}$ corresponds to annual basis functions and the last 30 rows correspond to the non-annual basis functions. In constructing the perturbation matrix $\boldsymbol{\epsilon}$, we take different strategy from that in Section 2. $\boldsymbol{\epsilon}$ also has size $36 \times 40$. We construct a non-normalized version of $\epsilon$ as follows,

$$
\begin{aligned}
& \boldsymbol{\epsilon}_{k j}=0, \text { if } k>6 ; \\
& \boldsymbol{\epsilon}_{k j}=0, \text { if } k \leq 6 \text { and } j=T_{k} ; \\
& \boldsymbol{\epsilon}_{k j} \sim i . i . d . \quad N(0,1), \text { otherwise. }
\end{aligned}
$$

Note that we add no perturbations on non-annual template functions. Then $\epsilon$ is normalized on each row. Next, we construct $\hat{\mathbf{C}}_{\alpha}$, the non-orthogonalized version of $\mathbf{C}_{\alpha}$ as $\hat{\mathbf{C}}_{\alpha}=\sqrt{1-\alpha} \mathbf{D}+$ $\sqrt{\alpha} \boldsymbol{\epsilon}$. Note the first 6 rows of $\mathbf{D}$ are orthogonal to corresponding rows of $\boldsymbol{\epsilon}$, which enables the first 6 rows of $\hat{\mathbf{C}}_{\alpha}$ to have unit length. Finally, $\mathbf{C}_{\alpha}$ is obtained by orthonormalizing $\hat{\mathbf{C}}_{\alpha}$. Then, we construct eigenfunctions as $\boldsymbol{\Gamma}_{\alpha}=\mathbf{C}_{\alpha} \boldsymbol{\varphi}$. In this simulation, we have 10 annual Fourier basis functions and form the reference space $\mathbf{F}_{10}$. To mimic our procedure for the Harvard Forest data, we retain the first $10 \mathrm{fPCs}$ to form $\boldsymbol{\Gamma}_{10}$. Then define eigenvalues associated with $\gamma_{k}^{\alpha}(t)$ as $\lambda_{k}=k^{-1.8}$. Choosing the exponent to be -1.8 makes the total variation explained by $\gamma_{10}$ to be $93.4 \%$, very close to the level in Harvard forest data. Consider a vector of $\alpha$ values $\left\{\alpha_{j}, 1 \leq j \leq E\right\}$. The $i$ th curve for $\alpha=\alpha_{j}$ is generated as

$$
x_{i}^{j}(t)=\sum_{k=1}^{36} s_{i j k}^{\gamma_{\alpha_{j}}} \gamma_{k}^{\alpha_{j}}(t), 1 \leq j \leq E, 1 \leq i \leq 400,
$$

where $s_{i j k}^{\gamma_{\alpha_{j}}}$,s are i.i.d. Gaussian distributed random variables with mean zero and variance $\lambda_{k}$. For the sake of computational efficiency, we have assumed the true curves to be directly observed and do not incorporate pre-smoothing into this simulation. For each $\alpha_{j}$, we construct 400 curves according to eigenstructure $\gamma_{\alpha_{j}}$. Then, we can perform the bootstrap hypothesis test on these 400 curves and achieve either rejection or acceptance of the null hypothesis. This test result can be described by a variable $w$ taking only values of 0 and 1 , with 0 corresponding to rejection and 1 corresponding to acceptance. We can repeat the above procedure for $B$ times, and record the value of $w$ at each time as $\left\{w_{b}, 1 \leq b \leq B\right\}$. Then the empirical power at $\alpha=\alpha_{j}$ is computed as $1-\sum_{b=1}^{B} w_{b} / B$.

In this simulation, we set $B=500$. The empirical power at a sequence of $\alpha$ values are computed. In presenting the power curve, we plot it not against $\alpha$ value but against the first correlation computed from $\gamma_{\alpha_{j}}$, denoted by $\rho_{1}^{\alpha_{j}}$; see Figure 6 . It is observed that $\rho_{1}^{\alpha}$ is a monotone decreasing function of $\alpha$. Moreover, the empirical size of the test is computed by setting $\alpha=0$; see Figure 6 . 

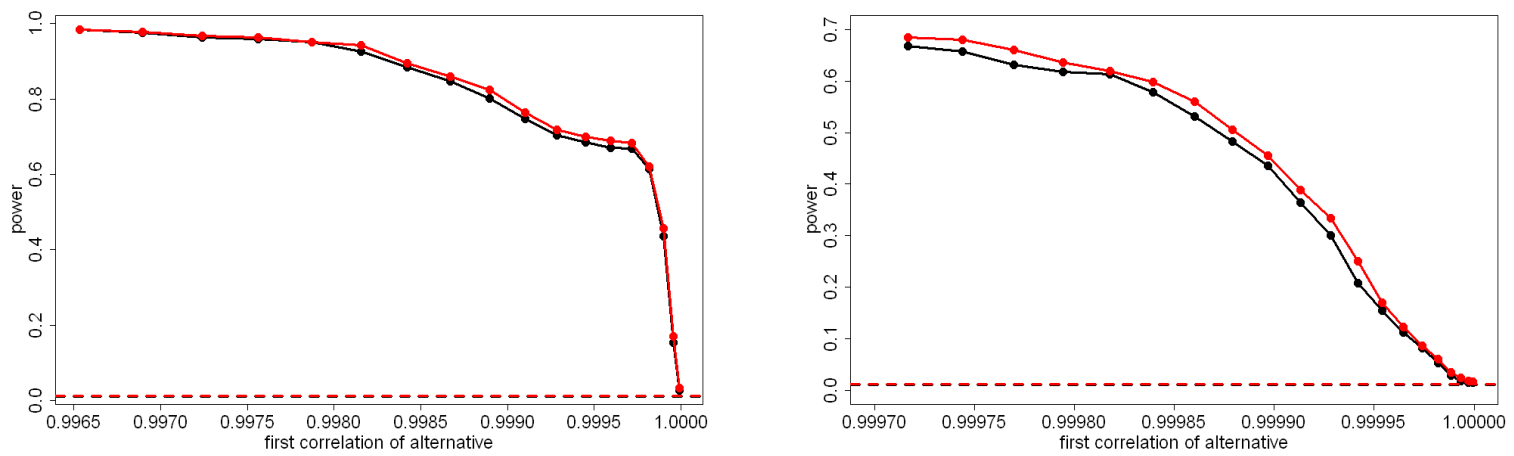

FiG 6 . In both plots, black curves and dashed lines represent the powers and the size (0.01) respectively derived by fixing number of $f P C$ s retained. Red curves and dashed lines represent the powers and the size (0.012) respectively derived by fixing percentage of variation explained. The horizontal axis is $\rho_{1}^{\alpha}$. Left: overall power curve; Right: power curve at the lower end

\section{References.}

Li, P.-L. and Chiou, J.-M. (2011). Identifying cluster number for subspace projected functional data clustering. Computational Statistics \& Data Analysis 55 2090-2103.

Yao, F., Müller, H.-G. and Wang, J.-L. (2005). Functional Data Analysis for Longitudinal Data. Annals of Statistics 33 2873-2903.

ZhANG, X., FriedL, M. and SChAAF, C. (2006). Global vegetation phenology from moderate resolution imaging spectroradiometer (MODIS): evaluation of global patterns and comparison with in situ measurements. Journal of Geophysical Research 111 G04017. 